\title{
A Method for Remote Control of EV Charging by Modifying IEC61851 Compliant EVSE Based PWM Signal
}

Pedersen, Anders Bro; Martinenas, Sergejus; Andersen, Peter Bach; Sørensen, Thomas Meier; Høj, Henning Si

\section{Published in:}

Proceedings of SmartGridComm 2015

Publication date:

2016

Document Version

Peer reviewed version

Link back to DTU Orbit

Citation (APA):

Pedersen, A. B., Martinenas, S., Andersen, P. B., Sørensen, T. M., \& Høj, H. S. (2016). A Method for Remote Control of EV Charging by Modifying IEC61851 Compliant EVSE Based PWM Signal. In Proceedings of SmartGridComm 2015 IEEE.

\section{General rights}

Copyright and moral rights for the publications made accessible in the public portal are retained by the authors and/or other copyright owners and it is a condition of accessing publications that users recognise and abide by the legal requirements associated with these rights.

- Users may download and print one copy of any publication from the public portal for the purpose of private study or research.

- You may not further distribute the material or use it for any profit-making activity or commercial gain

- You may freely distribute the URL identifying the publication in the public portal 


\title{
A Method for Remote Control of EV Charging by Modifying IEC61851 Compliant EVSE Based PWM Signal
}

\author{
Anders Bro Pedersen, Sergejus Martinenas, Peter Bach Andersen, Thomas Meier Sørensen, Henning Si Høj \\ Center for Electric Power and Energy \\ Department for Electrical Engineering \\ Technical University of Denmark \\ abp@elektro.dtu.dk
}

\begin{abstract}
The Electric Vehicle (EV) has properties that can not only load but can elevate its value as a resource to the power system. An EV represents a high-power, fast-responding flexible demand unit, with an attached energy storage (battery) and the potential for bi-directional capabilities (V2G). These properties can be used in a number of power and energy services, which can support power system operation while lowering the cost of EV ownership. Such services depend on the support from the communications technologies and standards that connect controllers and logic in the EVs with those in the charging infrastructure- and back-end systems. To date, there is still no widely adopted standard that supports EV grid services, such as smart charging. This work proposes a solution that would allow any combination of charging spot and EV - individually lacking the logic, communication and controllability required for smart charging - to gain this ability by simply adhering to the widely supported IEC61851 standard. This paper describes the solution, demonstrates the implementation and discusses the potential it has for unlocking EV power- and energy services.
\end{abstract}

Keywords-Electric Vehicle (EV), Electric Vehicle Supply Equipment (EVSE), Smart Charging, Remote Controlled Charging, Pulse Width Modulation (PWM), IEC61851

\section{INTRODUCTION}

With many nations around the world striving to increase the penetration of renewable energy resources in the electricity production, new problems and challenges arise. One of the most predominant is the inherent volatility of renewable energy sources such as wind and solar, the introduction of which causes instabilities in the production.

In Denmark, where large investments have been made in support of wind power, the penetration has long since exceeded $40 \%$. With such a large amount of renewable energy in the system, a lot of resources are being spent on providing balancing. Ever since the start of the recent wave of electric mobility, the EVs have been predicted to play an important role in the power system of tomorrow. With an ever increasing number of EVs being produced, their combined battery capacity, as well as their ability to instantly modify their prosumption profile, means they constitute a potentially massive untapped resource. Naturally, the EVs ability to participate in ancillary services, and thereby aid in facilitating a more stable power system, hinges on their availability throughout the day. Previous studies have been carried out in Denmark, which all point towards a round-the-clock availability in excess of $90 \%$, which supports the notion of having the EVs as a stable balancing resource [1][2].

Having the EVs plugged in is only part of the solution, since the individual vehicles have no knowledge of the degree of the balancing demand. In order to manage this response, a mechanism for remote controlling their charging is required. Thus far, a multitude of research projects have been carried out, mostly attempting to uncover the actual potential of the EVs alongside academic solutions for control algorithms [3][4][5]. Few projects, however, have offered anything in the direction of an actual control scheme for the vehicles [6][7]. To this day, no standards exists for controlling the smart charging of EVs, that have actually been put into mainstream production. The closest candidate is the proposed ISO15118 standard, which aims at offering EV-to-EVSE communication [8]. Where the initial version was mainly targeted at fleet- and charging point operators, and offered functions such as status- and the ability to identify the EV at the EVSEs, the latest version includes functions to support Vehicle-to-Grid (V2G) [9][10]. However, neither version specifies how this is to be implemented in the vehicles that do not have charging controllability as such.

The ISO15118 standard was created after- and based on a more basic standard called IEC61851, which is described in section II and which was originally designed to ensure a safe connection between EV and EVSE. ISO15118 was created to augment IEC61851. It enables high level communication between EV and EVSE by means Power Line Communication (PLC), where IEC61851 uses much simpler PWM and voltage level signaling [11]. Enabling ISO15118, which is still to be put into mainstream use, and will likely only be supported by very new and future EVs, requires a modification of all exiting and future EVSEs and EVs. On other hand, there is IEC61851, which is already implemented in all EVSEs and universally supported by existing EVs.

This paper describes a method for facilitating remote controllable charging in nearly all EVs on the market. It describes the general concept in section II, the implementation in the form of developed prototype in section III and the testing- and verification thereof in section IV. Lastly it concludes on the findings and the future work in ensuring the ideal- and stable operations of the prototype. 


\section{THE CONCEPT}

Past suggestions for controlling EV charging, have typically been relying on hardware being added to the vehicle, in a tightly coupled fashion. In order for this to work though, intricate knowledge of the vehicle's internal components is required, which makes any solution very specific to the individual EV. The advantage of the proposed solution, is that it works for nearly all EVs regardless of model or which EVSE it is connected to. This is accomplished by utilizing an existing EV standard, known as IEC61851.

\section{A. IEC61851}

Nearly all EVs that has rolled off the assembly line in the last half decade, support the standard called IEC61851 [12]. This standard was originally designed to ensure interoperability- and facilitate connection safety between EV and EVSE.

The first thing to happen when connecting an EV, is that the Plug Present (PP) pin makes contact. Between this pin and ground is a resistor, which specifies the rating of the cable. There are identical resistors in both ends of the cable, so both parties can see the rating. Next to the PP pin is the Control Pilot (CP) pin, which is used to communicate a series of states between the EV and the EVSE. The EVSE puts out a constant $12 \mathrm{~V}$. When a connection is made, a $2.7 \mathrm{k} \Omega$ resistor in the $\mathrm{EV}$ pulls this down to $9 \mathrm{~V}$, signaling that a safe connection has been established. There is an additional resistor on the EV side, rated at either 270 or $1.3 k \Omega$. This resistor is connected to a switch, which when closed signals that the EV is ready to charge. Depending on the value of the resistor, the power is further pulled down to either 3 or $6 \mathrm{~V}$ indicating whether or not the EV requires ventilation of the area during charging. The resistors can be seen in the EV- and EVSE side of figure 1 , respectively.
Having a safe connection is necessary before any charging can commence, but besides that, adhering to the various states and the built-in cable rating resistor, the EV is free to charge at any rate below that. Since this does not allow for enough control in such cases as grid congestion, the IEC61851 standard was extended to include a PWM signal, originating at the EVSE, which can be used to throttle the charging current. In reality, the signal sets the upper limit for the allowed charging power. Since nearly all Battery Management Systems (BMSs) in the EVs have charging algorithms designed to fill the battery as quickly as possible, the charging power throughout most of the charging session will be at the permitted maximum. Because of this, the limit essentially becomes a control signal. Since the PWM signal is a limit, the standard does not dictate that the EV should necessarily charge at the maximum rate, meaning that an EV charging at a lower rate than that set forth by the EVSE, is also considered normal behavior. That is especially the case in the beginning and the end of the charging process. The graph in figure 2 shows the charging current from 0 to $80 A$, in relation to the PWM duty cycle as specified by IEC61851.

\section{B. Modification of control pilot signal}

The concept described in this paper, can be regarded as a benign version of what, in the realm of computer networking, is referred to as a "man-in-the-middle" attack. The basis for "man-in-the-middle", is that an entity positions itself between two parties, acting towards both as if it was the other, making the parties believe they are communicating directly with each other. A Micro Controller Unit (MCU) reads the PWM signal generated by the EVSE and generates a new PWM signal towards the EV, which is remote controlled via GSM. The frequency remains the same, but in order to comply with IEC61851, the duty cycle of the generated PWM signal must not exceed that of the one read from the EVSE. This relationship between the duty cycles, can be expressed as follows:

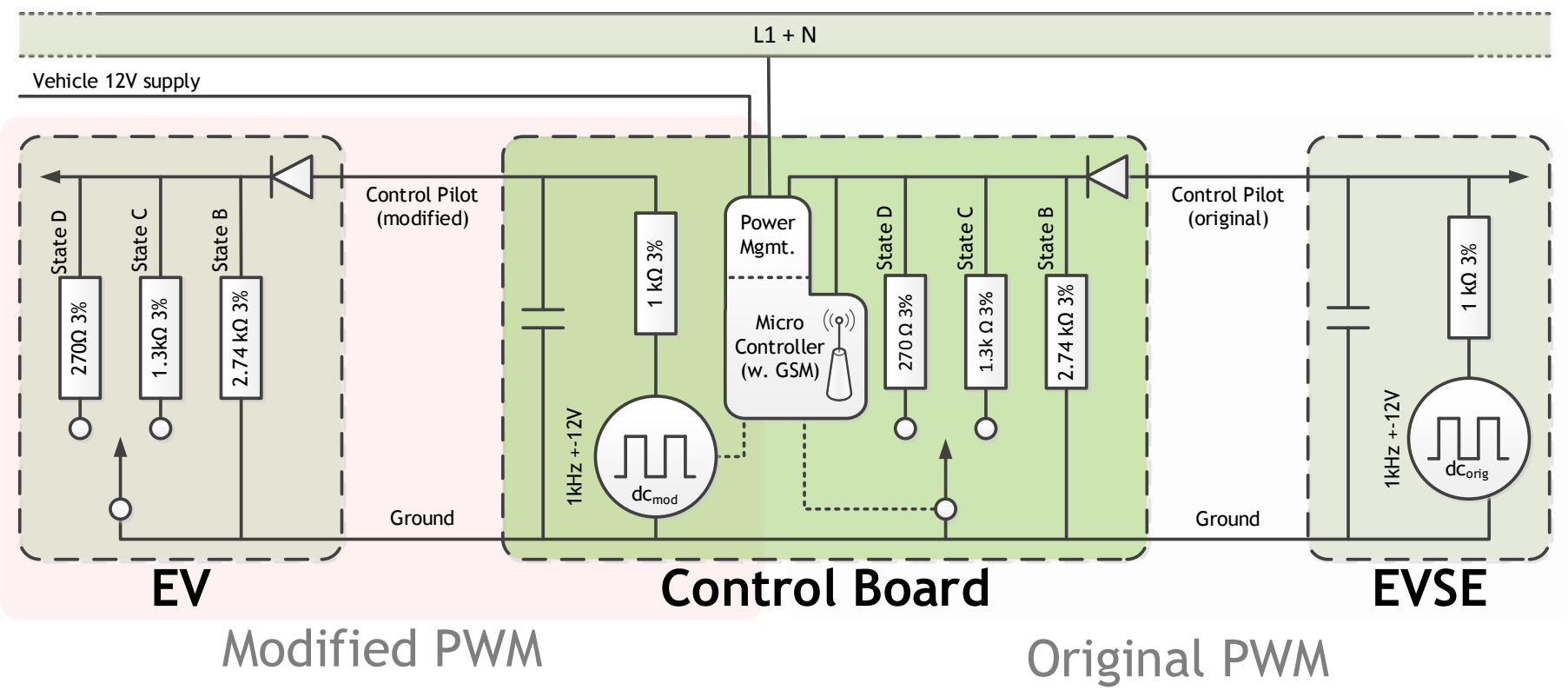

Fig. 1. The "man-in-the-middle" concept using a modified PWM signal 


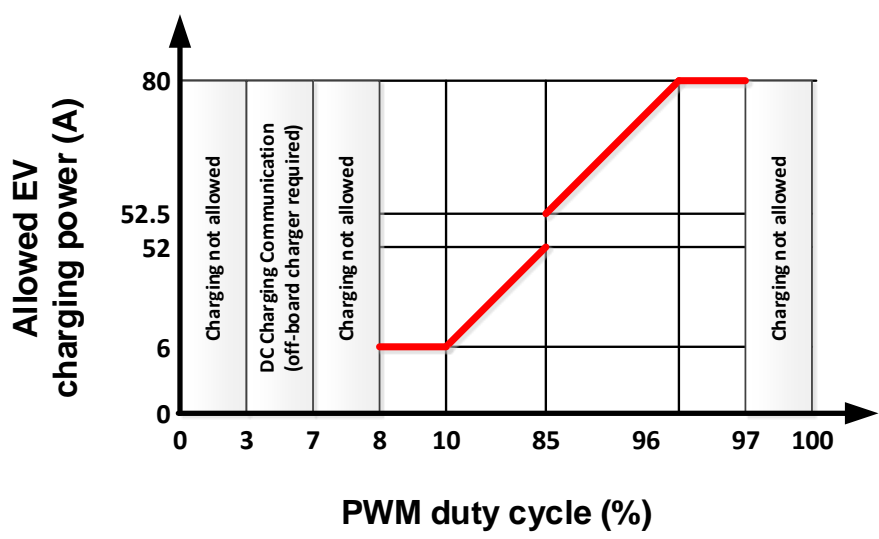

Fig. 2. Plot showing the relationship between duty-cycle and charge power, as specified by IEC61851

$$
D_{\text {mod }} \leq D_{\text {orig }}
$$

where $D$ is the duty cycle and mod and orig represent the modified- and original signals, respectively.

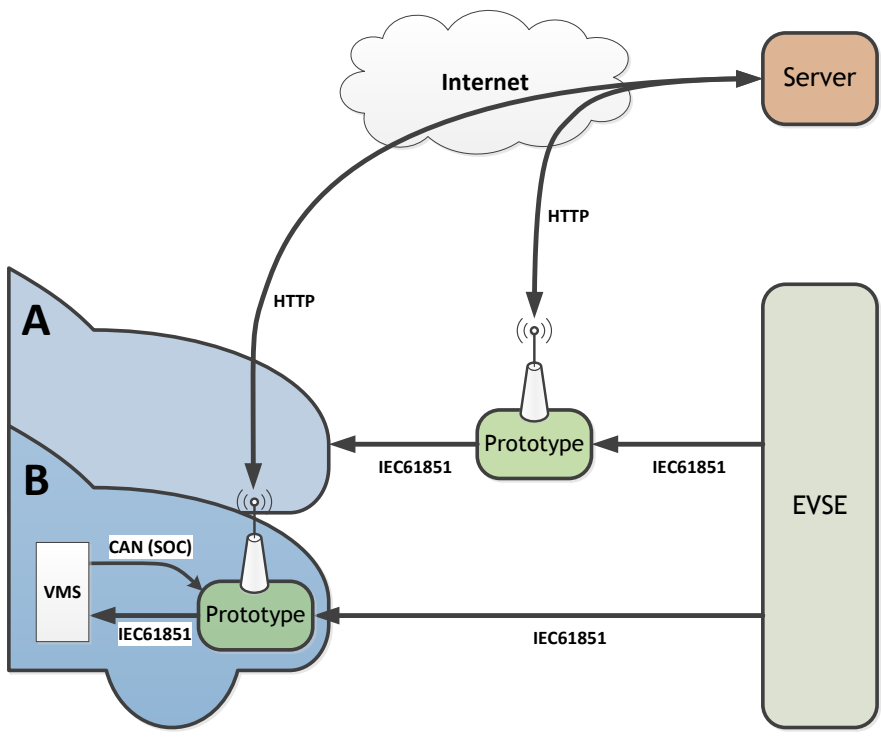

Fig. 3. Domain overview showing the prototype and the two proposed use cases, A) and B) (see text for description)

Figure 3 show an overview of the two main scenarios covered by the design: A) having the device in the actual charging cable and B) having it installed in the car. In both scenarios the PWM re-generation allows for the remote control of the charge power, but doing only this has limitations. In smart charging, meaning optimized charging where a certain energy goal is reached, the State Of Charge (SOC) needs to be known. Since this information is only available from within the car, the device would have to be mounted within reach of this. A proposed solution is to install the device immediately behind the charge connector in the EV, while connecting to the onboard Controller Area Network (CAN)-bus. Doing this gives read-access to, among others, the SOC. Assuming the proper plug adapter, this installation can be done in a relatively non-intrusive and easily reversible manner.

\section{Power}

Like all electronics, the board needs a power source in order to function. If placed in the car, as shown in figure 3 case " $\mathrm{B}$ " this is readily available. The $12 \mathrm{~V}$ system present in all cars, can easily supply a low-power Printed Circuit Board (PCB). If connected to the CAN bus via an On-board diagnostics (OBD) connector, which is the ideal data-source for getting the SOC, the $12 \mathrm{~V}$ is also available through this. Other projects at the Technical University of Denmark (DTU) have successfully done exactly this. For example, a Danish GAP funded project, also run by DTU, created a Global Positioning System (GPS)- and CAN based datalogger [13]. Borrowing from the experiences learned in this project, not only provided knowledge about the CAN connection, but also the codes associated with reading the SOC.

In the other of the two cases, shown in figure 3 case "A" with the board situated in the charging cable, the power becomes a bit of a challenge. Because the $12 \mathrm{~V}$ system of the vehicle is not available, an alternative power source would be required. Tests are still being carried out, but it is the consensus that with a sufficiently low-powered MCU the board could potentially be run off the $12 \mathrm{~V}$ PWM CP signal coming from the EVSE. Given that the primary application for the in-the-cable solution is regulating services, the need for fast communication is even higher than for regular smart charging. This increases the power requirements, since the GSM modem is by far the component requiring the most power. On the other hand, regulating services are typically offered during charging, so with power flowing through the cable at a minimum of $6 A$ (see figure 2), another alternative could be an inductive solution. The board would then start in a low-power mode, with only the MCU running at first, powered by the CP signal. When power starts flowing, the induction power source would take over, and the Global System for Mobile Communications (GSM) connection could be established and regulating could begin. The $12 \mathrm{~V}$ connection to the vehicle, along with the thought inductive power tap is illustrated in figure 1 .

\section{PROTOTYPE}

As part of the EV research activities at DTU, as well as to test- and verify the feasibility of the concept, work on a prototype has been carried out in the spring of 2015. The first version was finished in May, and tested shortly thereafter (see section IV), the second prototype with a smaller footprint has been finished in the summer of 2015 .

\section{A. $P C B$ design}

Both the prototypes were realised as single PCBs. The second prototype, with a smaller footprint, is shown in figure 4. Goals for the design included the implementation of the full functionality of the concept, a small footprint and comfortable testing; the latter meaning easy test-point access and support for easier hardware debugging. The first prototype board has a size of $10 \times 10 \mathrm{~cm}$, the second is half the size. The board has connections for $12 \mathrm{~V}$ power input, a $\mathrm{CP}$ line for the $\mathrm{EV}$ - and EVSE side as well as the connection for vehicle CAN bus. 
The main parts of the design are:

- Main MCU circuit

- $\quad$ PWM and EV state mirroring circuit

- Wireless communication circuit

- Power supply circuit

The main MCU takes care of measuring and generating the PWM signal. The PWM and EV state mirroring circuit consists of two parts: the measurement circuit and the signal boosting circuit. The PWM signal from the EVSE CP line is converted from a $12 \mathrm{~V}$ level to a level measurable by the MCU, using voltage division in the measurement circuit. This circuit also includes the resistors to mirror the EV states. The new PWM signal is generated by the MCU and then boosted to $12 \mathrm{~V}$ using transistors. The wireless communication circuit consists of a GSM modem with a power supply circuit and a SIM card. The power supply circuit converts $12 \mathrm{~V}$ input power to multiple power levels for different devices on the board. The second version of the board includes the GSM chip with integrated GPS.



Fig. 4. Prototype board version 2.0 (codename CHILI)

The PCB was designed with a feature, allowing the original PWM signal to bypass the device and go straight through to the EV. This way, when the board is powered off, it is not interrupting the normal charging of the EV.

\section{B. Communication}

In order to test the remote controllability of the prototype, a rudimentary communication channel was created based on the HyperText Transport Protocol (HTTP) protocol. Any commands for the board to execute, are submitted to a webserver using HTTP POST, which then logs the command. As part of its operating cycle, the prototype then performed an HTTP GET request for the latest command to execute. In the same GET request, various debugging information is included, such as the PWM duty cycle read from the EVSE and various operating states. This is illustrated in figure 5 as two separate connections, going from the prototype to the server. These data are, like the commands, logged on the webserver for future use in testing/debugging. Because the prototype is based on an Atmel AVR MCU, which has no built in support for threading, the communication has to be performed in-line with the remaining functions. Currently some of these functions are implemented with generous delays to ensure stable timing when interfacing with other components, which slows the cycle time between each HTTP request to between 5 and 7 seconds.

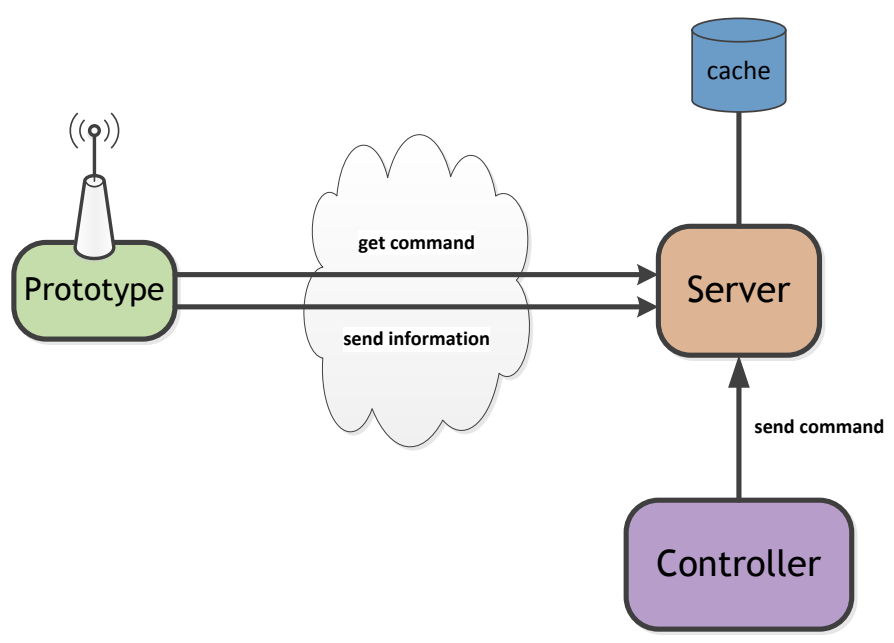

Fig. 5. Communication overview

Because a standard GSM connection receives a dynamic IP by the Internet Service Provider (ISP) as well as being subject to Network Address Translation (NAT), which effectively isolates the client and limits it to establish outbound connections, it was easier to have the client establish connections to the server. An HTTP based solution was chosen for its simplicity, but a raw socket based solution has been considered, if HTTP cannot be optimized enough. Since this would require custom server software to be written, this is planned for the next iteration prototype.

While the primary concept outlined in this paper is the board, facilitating remote controlled charging by modifying the PWM, a couple of words should be said about both scalability and security.

1) Scalability: Regardless of the specific communication protocol, the most likely scenario is one where the boards connect to the server. Currently, with HTTP, the setup is stateless resulting in the server not having to support a large number of the connections. Any requests made by the boards, would just be queued up and served one at a time. Depending on the speed at which the commands should be fetched and executed, the webserver would have to be optimized to accept more concurrent connections. The alternative, where the boards establish and maintain a socket connection to the server, has the advantage of a short response-time. This does, however, come at the cost of having to maintain a socket connection for each connecting client.

Currently the setup uses a MySQL database server, which is shown in figure 5 as a cache, to store the commands to be retrieved by the boards. Depending on optimization, an inmemory caching server could be used instead, to buffer the commands. The information sent from the board would still go into the database.

2) Security: Most any protocol that could be used to communicate between board and server could- and should be 
encrypted to avoid tampering. Both HTTP and raw sockets support this through the use of Secure Sockets Layer (SSL) and Transport Layer Security (TLS). Since this is tried and tested in equally vital applications such as banking, there is little reason to consider it unsuitable for smart grid applications. Moreover, the GSM chip also supports HTTP over SSL (HTTPS).

As important as securing the data transfer, is to establish the identity of the board, since the charging response could be highly location sensitive. In the test setup, the International Mobile station Equipment Identity (IMEI) number from the SIM card was used to uniquely identify the board. If the IMEI is not trustworthy, a safer alternative would be a pre-shared key/token.

\section{TESTING}

Part of the requirements for the prototype, is that it follows the IEC 61851 standard. In order to verify this, a series of test cases was devised. Of the most important were to a ensure safe and comfortable charging process, with operation of the device in various conditions, verifying that:

1) The prototype can mirror the EV states to the EVSE

2) The output PWM can mirror the input PWM

3) The output PWM can be remote controlled

4) The generated PWM duty cycle is not exceeding the measured one (see section II)

All these test cases were carried out using the test setup based on the use case "A" shown on figure 3 and previously discussed in section II, when the device is placed in the cable. There the Original Equipment Manufacturer (OEM) EV is connected to an actual $16 \mathrm{~A}$ capable EVSE using a standard charging cable. The charging cable has the $\mathrm{CP}$ line cut and the device inserted.

Firstly the proper functioning of the charging process was checked. The device correctly detected the EV states and mirrored them towards the EVSE. That is confirmed by the successful initiation of EV charging process.

Secondly, the PWM signal was also correctly measured and reproduced on the EV side. This can be observed in figure 6 regions marked red. It shows the measured EV state together with the duty cycles of the measured- and the generated PWM signals on the CP line. There, measured (green curve) and generated (blue curve) signals match at $26 \%$ duty cycle, and confirmed by the EV charging at $16 \mathrm{~A}$ as expected.

Thirdly, after the normal functioning of the device was confirmed, the modification of the original EVSE PWM signal was tested. The original PWM signal duty cycle coming from the EVSE is around $26 \%$, which corresponds to maximum $16 \mathrm{~A}$ charging current, this is shown in figure 6 regions marked blue. This duty cycle was modified and set to $20 \%$ which corresponds to $12 \mathrm{~A}$ charging current and then to $10 \%$ which corresponds to $6 \mathrm{~A}$. All the commands took around $10 \mathrm{~s}$ to execute, due to large debugging delays on the data polling from GSM modem.

Fourthly, to test the function of the device to not exceed the PWM signal from the EVSE, the generated PWM signal was returned to original value of $26 \%$. Afterwards the signal coming from the EVSE was set to a value of $15 \%$, corresponding


Fig. 6. EV states with measured and generated PWM signal duty cycles

to $10 \mathrm{~A}$ charging current. It can be seen in the figure 6 , that the device has followed the changes coming from the EVSE and correctly responded to the restriction, regions marked green.

Lastly, a small anomaly is observed in the middle of figure 6. While the measured and generated PWM signals are staying at $26 \%$, charging current drops from $16 A$ to $0 A$ for a second and comes back to $16 \mathrm{~A}$. This behavior has been previously observed with this combination of the EV and EVSE, while they were directly connected. Therefore it is concluded that this behavior is most likely coming from the EV.

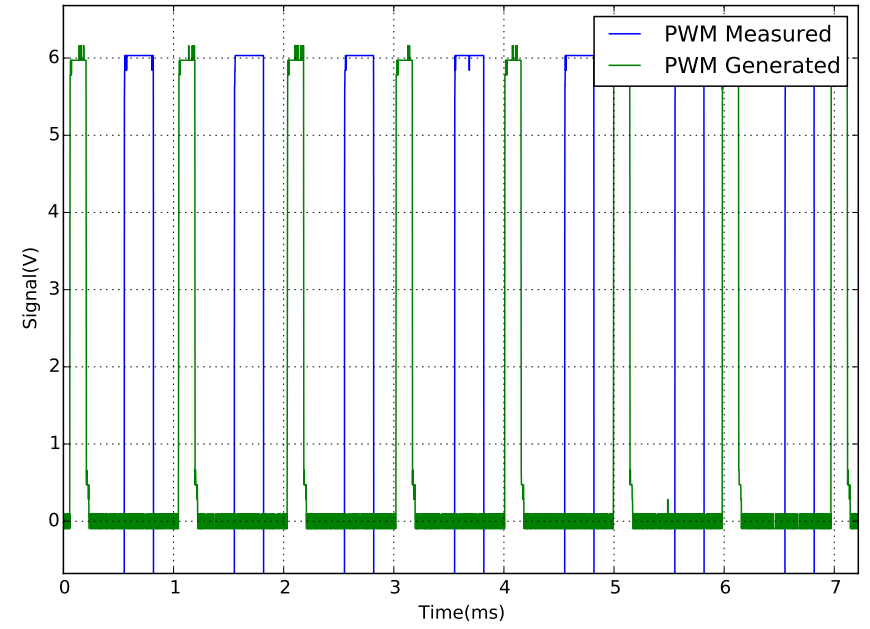

Fig. 7. Measured and generated PWM signal

Figure 7 shows the measured and generated PWM signals. It is clearly seen that the generated signal (green curve) has a lower duty cycle of $15 \%$ than the measured from the EVSE (blue curve) at $26 \%$. It should be noted that the generated signal only produces the positive side PWM, that is due to 
the fact that the $\mathrm{CP}$ line sensing circuit, in the EV, includes the diode to cut the negative side of the PWM anyway. Future versions of the board will however reproduce the PWM signal positive and negative side, to completely fulfill IEC61851 specification.

\section{ACKNOWLEDGEMENTS}

The tests carried out to verify the proper operation of the prototype, were carried out in the Electric Vehicle Lab, which is one of the laboratories under PowerLab.dk, which is again part of the Center for Electric Power and Energy (CEE) at DTU Elektro [14] .

Part of the test setup included custom made control equipment, which works as stand-ins for either the EVSE, the cable or the EV. Since most OEM EVSEs and EVs do not allow control of either PWM or internal states, this equipment was invaluable in verifying the prototype.

This test equiment was originally developed during the Danish Nordic Electric Vehicle Interoperability Center (NEVIC) project, and is currently used by the NEVIC [15].

The brand new Nissan Leaf used in the experimental setup, was on loan from Nissan Denmark, who had supplied it for research purposes in relation to the NIKOLA EV research project [16].

\section{CONCLUSION}

In order for the EVs to help facilitate a more stable electricity system, with an ever increasing penetration of renewables, their charging needs to be controllable. Historically this control has not been a focus of the OEMs and to a large extent continues not to be.

The proposed solution to remotely modify the PWM signal set by the EVSE set, in accordance with the IEC61851 standard, represents a novel way of introducing controllable charging without the need to modify neither the EV nor the EVSE. If additional control is desired, like the ability to perform smart charging, the SOC can be accessed from the onboard systems of the vehicle without making any permanent changes.

The developed prototype demonstrated the feasibility of the concept, allowing the PWM signal from the EVSE to be read and either mirrored- or modified towards the EV. While tests have been carried out to verify that the PWM control does in fact work, and that the board can mimic the states to the extend that is required to facilitate the charging, further tests are needed in order to ensure that all possible states can be mirrored.

\section{A. Future works}

While several tests have already been performed to verify the prototype (see section IV), further testing is still required before the board can safely be put into permanent use. Least of all is proper enclosure, to ensure dust and/or weather sealing, but a complete test of all states and extremes are still outstanding. Most of these test are planned for the early- to mid summer of 2015.
The first version of the prototype made use of an HTTP based polling mechanism, where a webserver is used to sync request. The simplicity of the scheme as well as the implementation thereof, resulted in a 5-7 second response time. Since this is sufficient for many ancillary services and definitely smart charging, the board was deemed fast enough. Alternatives for an improved communication model is currently being investigated, and the next iteration will likely see a significant improvement in the control-cycle time.

Disclaimer: The proposed design was submitted to the European patent office in the fall of 2014 and is currently pending final approval.

\section{REFERENCES}

[1] A.B. Pedersen, A. Aabrandt, J. Østergaard, B. Poulsen, Generating Geospatially Realistic Driving Patterns Derived From Clustering Analysis Of Real EV Driving Data, IEEE ISGT Asia, 2014.

[2] Q. Wu, A.H. Nielsen, J. Østergaard, S.T. Cha, F. Marra, Y. Chen, C. Træholt, Driving Pattern Analysis for Electric Vehicle (EV) Grid Integration Study, IEEE ISGT Europe, 2010.

[3] K. Knezovic, M. Marinelli, R.J. Møller, P. B. Andersen, C. Træholt, F. Sossan, Analysis of Voltage Support by Electric Vehicles and Photovoltaic in a Real Danish Low Voltage Network, IEEE UPEC, 2014.

[4] K. Knezovic, M. Marinelli, P.B. Andersen, C. Træholt, Concurrent Provision of Frequency Regulation and Overvoltage Support by Electric Vehicles in a Real Danish Low Voltage Network, IEEE IEVC, 2014.

[5] A. Di Giorgio, F. Liberati, S. Canale IEC 61851 compliant electric vehicle charging control in smartgrids, 21st Mediterranean Conference on Control and Automation, 2013

[6] S. Martinenas, M. Marinelli, P.B. Andersen, C. Træholt, Implementation and Demonstration of Grid Frequency Support by V2G Enabled Electric Vehicle, , IEEE UPEC, 2014.

[7] S. Martinenas, A.B. Pedersen M. Marinelli, P.B. Andersen, C. Træholt, Electric Vehicle Smart Charging using Dynamic Price Signal, IEEE IEVC, 2014.

[8] ISO15118 standard

[9] M. Multin, F. Allerding, H. Schmeck Integration of electric vehicles in smart homes - an ICT-based solution for V2G scenarios, IEEE ISGT, 2012

[10] J. Schmutzler, C. Wietfeld, C.A. Andersen Distributed energy resource management for electric vehicles using IEC 61850 and ISO/IEC 15118, IEEE Vehicle Power and Propulsion Conference, 2012

[11] C. Lewandowski, S. Gröning, J. Schmutzler, C. Wietfeld Interference analyses of Electric Vehicle charging using PLC on the Control Pilot, IEEE International Symposium on Power Line Communications and Its Applications, 2012

[12] IEC61851 standard

[13] M. Svendsen, M. Winther-Jensen, A.B. Pedersen, P.B. Andersen, T.M. Sørensen, Electric vehicle data acquisition system, IEEE IEVC, 2014.

[14] http://www.powerlab.dk/

[15] http://www.cee.elektro.dtu.dk/research/NEVIC

[16] P.B. Andersen, M. Marinelli, O.J. Olesen, C.A. Andersen, G. Poilasne, B. Christensen, O. Alm, The Nikola project - Intelligent Electric Vehicle Integration, IEEE ISGT Europe, 2014. 